\title{
Diagnostic and therapeutic decisions of general practitioners in patients with suspected or diagnosed non-alcoholic fatty liver disease with reference to current European guidelines
}

\author{
Magdalena Olszanecka-Glinianowicz'1 Jerzy Chudek $^{2}$ \\ ${ }^{1}$ Health Promotion and Obesity Management Unit, Department of Pathophysiology, Medical Faculty in Katowice, \\ Medical University of Silesia, Katowice, Poland \\ ${ }^{2}$ Department of Internal Medicine and Oncological Chemotherapy, School of Medicine in Katowice, Medical University \\ of Silesia, Katowice, Poland
}

\begin{abstract}
Objective: The aim of the multicenter study was to assess the compliance of diagnostic and therapeutic decisions of general practitioners in patients with suspected or diagnosed non-alcoholic fatty liver disease (NAFLD) with current guidelines of the European Association for the Study of the Liver, the European Association for the Study of Diabetes and the European Association for the Study of Obesity.

Material and methods: The multicenter survey was performed nation-wide by 844 general practitioners among 20,550 outpatients suspected for or already diagnosed with NAFLD (48.2\%) during a routine visit. The lack of oral consent to participate and the inability to obtain answers to the survey questions were the only exclusion criteria.

Results: In the group suspected for NAFLD, activity of liver enzymes and ultrasound were ordered in $48.3 \%$ and $54.7 \%$ of patients. Among non-diabetic patients already diagnosed with NAFLD fasting glucose level was measured in $75.7 \%$, an oral glucose tolerability test was performed in $38.7 \%$, fasting insulin level was measured in $15.1 \%$, and HOMA-IR was calculated in 5.5\%. In the therapy the following were recommended: reduction of diet energy by $500-1000 \mathrm{kcal}$ corresponding to the patient's needs (in $40.9 \%$ and $87.2 \%$ suspected and diagnosed with NAFLD, respectively), restriction of the consumption of animal fat (in $45.1 \%$ and $93.7 \%$ ) and alcohol (in $39.2 \%$ and $80.7 \%$ ), increase in the consumption of complex carbohydrates (in $28.6 \%$ and $62.9 \%$ ), avoidance of drinks and foods rich in fructose (in 32.4\% and 71.5\%), regular aerobic physical activity 150-200 minutes per week (in 39.2\% and $82.5 \%$ ) and regular resistance training (in 14.4\% and 31.5\%). Pharmacological treatment of concomitant diseases was prescribed in $38.7 \%$ and $73.4 \%$ of patients, respectively, including pharmaceutical products containing the necessary phospholipids (in 13.6\% and 36.0\%) and thiazolidine carboxylic acid (in 29.2\% and 74.9\%).
\end{abstract}

Conclusions: 1 . Polish general practitioners too rarely perform a diagnostic test for NAFLD and recommend changes in diet and physical activity, and too rarely perform tests for carbohydrate metabolism disturbances. 2. Diagnostic workup and NAFLD therapy are in line with the current guidelines.

Key words: NAFLD, diagnosis, treatment, compatibility with guidelines.

\section{Introduction}

Non-alcoholic fatty liver disease (NAFLD) occurs in $17-33 \%$ of the population of developed countries. About $1 / 3$ of this population develops non-alcoholic steatohepatitis (NASH) and 5-year risk of development of liver cirrhosis among patients with NASH is $15 \%[1,2]$. The frequency of NAFLD increases significantly among obese subjects $(70-80 \%$ of this population) [3]. Visceral fat mass increase of $1 \%$ causes $40 \%$ increase in the accumulation of lipids in the hepatocytes, while the increase in total fat mass or subcutaneous fat mass by $1 \%$ raises he-

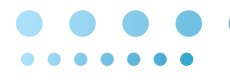


patic lipid accumulation by $20 \%$ [4]. However, NAFLD may also develop in the course of hyperlipidemia, lipodystrophy, congenital disorders of lipoprotein metabolism, Anderson's disease, Weber-Christian syndrome, Mauriac syndrome, Wilson's disease, celiac disease, inflammatory bowel disease and during parenteral nutrition and starvation. It should be noted that NAFLD induced by obesity is a primary form of these diseases, while development in the course of other diseases is secondary.

The known risk factors of NAFLD development include high-energy diet, excessive intake of saturated fat and refined carbohydrates, and high consumption of sugared beverages with sugar and fructose [5].

In accordance with the European Association for the Study of the Liver, the European Association for the Study of Diabetes and the European Association for the Study of Obesity guidelines from 2016 in patients with obesity, insulin resistance, carbohydrate metabolism disturbances, dyslipidemia and hypertension a screening test for NAFLD assessing the excess of fat in the liver should be performed. In subjects with secondary NAFLD causes, alcohol consumption should be excluded. The concomitant diseases which may increase liver damage should also be diagnosed [6]. In addition, during screening for NAFLD diet and physical activity level should be assessed. In all patients with hepatic steatosis regardless of liver enzymes activity components of metabolic syndrome should be diagnosed. All subjects with persistent elevated liver enzymes should be tested for NAFLD. In subjects with components of metabolic syndrome a screening test for NASH such as liver enzymes determination and/or liver ultrasound should be performed. Finally, in high-risk groups (age $>50$ years, type 2 diabetes and/ or other components of the metabolic syndrome), active detection of NASH with fibrosis is recommended [6].

In primary health care liver steatosis should be diagnosed using ultrasound. Non-invasive methods for assessing fibrosis can be reliably used in initial stratification of risk to rule out a severe form of the disease [6].

In patients with NAFLD without type 2 diabetes, insulin resistance assessment based on HOMA-IR and a screening test for type 2 diabetes should be performed. In patients with type 2 diabetes should be screened for NAFLD regardless of liver enzymes activity [6].

The complex assessment of patients with suspected NAFLD should include: alcohol consumption, occurrence in a patient or in the family history of type 2 diabetes, hypertension and cardiovascular diseases, body mass, height and waist circumference measurements and body mass index (BMI) calculation, analysis of body mass changes, occurrence of hepatitis $B$ virus (HBV) and hepatitis $\mathrm{C}$ virus ( $\mathrm{HCV}$ ) infections, the use of drugs that can cause steatosis, determination of liver enzymes activity, measurement of fasting glucose and insulin levels and
HOMA-IR calculation as well as oral glucose tolerance test (OGTT), determination of morphology, lipid profile and uric acid concentration and liver ultrasound [6].

In all patients with NAFLD, fibrosis should be assessed on a point scale based on serum markers (such as NFS, FIB-4, ELF or FibroTest) in order to exclude significant fibrosis ( $\geq$ F2). If significant fibrosis cannot be ruled out, the patient should be referred to the hepatology clinic for elastography. If significant fibrosis is confirmed, a biopsy should be performed [6].

The basis of NAFLD treatment is changes in eating habits such as reduction of diet energy by 500-1000 kcal in relation to individual needs to obtain $7-10 \%$ weight reduction, reducing the consumption of saturated fat and increasing the consumption of complex carbohydrates, avoiding drinks and foods rich in fructose and restriction of alcohol consumption. The second basic element of treatment is regular aerobic physical activity 150-200 minutes per week with moderate intensity and resistance training. In addition, pharmacological treatment of obesity complications should be recommended. There are currently no drugs registered for the treatment of NASH [6]. The data concerning efficacy of essential phospholipids, which are glycerol esters of choline phosphoric acid and unsaturated fatty acids - linolenic, linolenic and oleic - and thiazolidine carboxylic acid come from experimental studies and their efficacy in humans is still poorly documented $[7,8]$.

The aim of the multicenter study was to assess the compliance with diagnostic and therapeutic decisions of Polish general practitioners in patients suspected for or diagnosed with NAFLD with current guidelines of the European Association for the Study of the Liver, the European Association for the Study of Diabetes and the European Association for the Study of Obesity.

\section{Material and methods}

The multicenter survey was performed nation-wide by 844 Polish general practitioners. These doctors interviewed 20,550 patients referred to outpatient clinics. The criterion for inclusion in the study was the application to the clinic, and the exclusion criteria were lack of oral consent to participate in the study and the inability to obtain answers to the questions contained in the survey.

The survey included anthropometric data (body mass, height, BMI and waist circumference) and two blood pressure measurements taken during the visit; sociodemographic data [age, sex, education (primary/vocational/secondary/higher/not applicable), place of residence (village/city < 50,000 residents/city 50,000-200,000 residents/city $>200,000$ residents)] and clinical data [diagnosis being the reason for reporting to the clinic (obesity/ diabetes type 1 /diabetes type 2 /hypertension/dyslipide$\mathrm{mia} /$ ischemic heart disease/heart failure/chronic hepatic 
disease/other), other chronic disease occurred in patients (obesity/diabetes type 1/diabetes type 2/hypertension/ dyslipidemia/ischemic heart disease/ heart failure/chronic hepatic disease/other), used drugs (ACE-I, sartan, loop diuretic, thiazide diuretic, thiazide diuretic, statin, fibrate, ezetimibe, metformin, sulphonylurea derivative, insulin, other), previous diagnosis of NAFLD (determination of liver enzymes/liver ultrasound/ point scale/elastography/ other), the interview during this visit in subjects with and without previous diagnosis including: alcohol consumption/occurrence in patient or in family history of type 2 diabetes, hypertension and cardiovascular diseases/changes of body mass - significant gain or loss in short period of time/a history of HBV and HCV infections as well as commissioning tests such as: liver enzymes activity/liver ultrasound, diagnosis of NAFLD in patients who have been diagnosed before, excluding other causes of liver steatosis, performance in a patient without diagnosed diabetes: fasting glucose/fasting insulin/OGTT/HOMA-IR calculation/morphology/lipid profile/urid acid concentration; assessment of fibrosis based on a point scale based on serosal markers in patients diagnosed with NAFLD; referral of a patient in whom fibrosis cannot be ruled out to the hepatology clinic; performed in a hepatology clinic: elastography/biopsy of the liver; treatment procedures implemented in a patient diagnosed with NAFLD and with suspected NAFLD (reduction of diet energy by $500-1000 \mathrm{kcal}$ in relation to the patient's current demand/limitation of consumption of animal fats/increased consumption of complex carbohydrates/avoidance of fructose-rich beverages and foods/restriction of alcohol consumption/regular aerobic physical activity 150-200 minutes per week/ regular resistance training/pharmacological treatment of concomitant diseases/used of preparation containing the necessary phospholipids/used of preparation containing thiazolidine carboxylic acid/others)].

Statistical analysis was performed with Statistica 12.0 software (Cracow, Poland). Values of variables were presented as percentages and the mean values with standard deviations (SD). The groups were compared using the $\chi^{2}$ and Student's $t$ tests. The value of $p<0.05$ was considered to be statistically significant.

\section{Results}

The demographic and clinical characteristics of the study groups are presented in Table 1.

Before inclusion in the study diagnosis of NAFLD was established in $48.2 \%$ of study subjects, most often among patients referred to the visit due to ischemic heart disease (56.7\%) and type 2 diabetes (53.3\%) and the least due to obesity (45.3\%). Most frequently the diagnostic workup included determination of liver enzymes (96.7\%) and liver ultrasound (86.4\%). Among subjects screened previously
NAFLD was diagnosed in $47.8 \%$ and in $70.5 \%$ with NAFLD other causes of fatty liver disease were excluded (Table 2).

During the current visit, in the group of patients not screened earlier, the physicians obtained data concerning: alcohol consumption (in 91.2\%), occurrence of type 2 diabetes in the patient and his family (in 90.8\%), recent significant weight loss or gain in a short period of time (in 87.7\%), a history of HBV and HCV infection (in 76.3\%). In addition, physicians ordered assessment of the activity of liver enzymes in $54.7 \%$, and liver ultrasound in $48.3 \%$ (Table 2).

In patients with NAFLD without diabetes the fasting glucose level was determined in $75.7 \%$, an oral glucose tolerability test was performed in $38.7 \%$, the fasting insulin level was measured in $15.1 \%$ and HOMA-IR was calculated in $5.5 \%$. In addition, among patients with NAFLD physicians determined total blood count in $71.1 \%$, lipid profile in $66.4 \%$ and uric acid concentration in $65.2 \%$ (Table 2).

In only $21.5 \%$ of patients diagnosed with NAFLD liver fibrosis was estimated using a point scale based on serous markers and $61.3 \%$ of patients in whom significant fibrosis could not be excluded were referred to a hepatology clinic. In patients referred to a hepatology clinic elastography and liver biopsy were performed in $40.1 \%$ and $13.2 \%$, respectively (Table 2 ).

In accordance with guidelines doctors recommended to the patients:

- reduction of diet energy by 500-1000 kcal below the patient's needs in $87.2 \%$ of patients with NAFLD and in $40.9 \%$ suspected for NAFLD,

- restriction of animal fat consumption in $93.7 \%$ of patients with NAFLD and $45.1 \%$ suspected for NAFLD,

- increase in the consumption of complex carbohydrates in $62.9 \%$ of patients with NAFLD and $28.6 \%$ suspected for NAFLD,

- avoiding drinks and foods rich in fructose in $71.5 \%$ of patients with NAFLD and $32.4 \%$ suspected for NAFLD,

- restriction of alcohol consumption in $80.7 \%$ of patients with NAFLD and 39.2\% suspected for NAFLD,

- regular aerobic physical activity (150-200 minutes per week) in $82.5 \%$ of patients with NAFLD and $39.4 \%$ suspected for NAFLD,

- regular resistance training in $31.5 \%$ patients with NAFLD and $14.4 \%$ suspected for NAFLD,

- pharmacological treatment of concomitant diseases in $73.4 \%$ of patients with NAFLD and $38.7 \%$ suspected for NAFLD (Table 3).

Doctors' prescription not included in the assessed guidelines:

- pharmaceutical products containing the necessary phospholipids in $36.0 \%$ of patients with NAFLD and $13.6 \%$ suspected for NAFLD, 
Table 1. Characteristics of study group [ $N=20,550]$

\begin{tabular}{|c|c|c|c|c|}
\hline Parameters & $\begin{array}{l}\text { Study group } \\
(N=20,550)\end{array}$ & $\begin{array}{c}\text { Women } \\
(n=10,035)\end{array}$ & $\begin{array}{c}\text { Men } \\
(n=10,515)\end{array}$ & $p$ \\
\hline \multicolumn{5}{|l|}{ Demographic data } \\
\hline Age [years] & $55 \pm 11$ & $56 \pm 11$ & $54 \pm 12$ & NS \\
\hline \multicolumn{5}{|l|}{ Education level } \\
\hline Primary $[n ; \%]$ & 1,$229 ; 6.0$ & $706 ; 7.0$ & $523 ; 5.0$ & \multirow{4}{*}{ NS } \\
\hline Vocational [n; \%] & 5,$630 ; 27.4$ & 2,$429 ; 24.2$ & 3,$201 ; 30.4$ & \\
\hline Secondary $[n ; \%]$ & 8,$911 ; 43.4$ & 4,$608 ; 45.9$ & 4,$303 ; 40.9$ & \\
\hline Higher $[n ; \%]$ & 4,$780 ; 23.3$ & 2,$292 ; 22.8$ & 2,$488 ; 23.7$ & \\
\hline \multicolumn{5}{|l|}{ Place of residence } \\
\hline Rural [ $n$; \%] & 4,$646 ; 22.6$ & 2,$314 ; 23.1$ & 2,$332 ; 22.2$ & \multirow{4}{*}{ NS } \\
\hline City $<50,000$ residents $[n ; \%]$ & 4,$041 ; 19.7$ & 1,$894 ; 18.9$ & 2,$147 ; 20.4$ & \\
\hline City $50,000-200,000$ residents [ $n$; \%] & 6,$266 ; 30.5$ & 3,$114 ; 31.0$ & 3,$152 ; 30.0$ & \\
\hline City > 200,000 residents [ $n$; \%] & 5,$597 ; 27.2$ & 2,$713 ; 27.0$ & 2,$884 ; 27.4$ & \\
\hline \multicolumn{5}{|c|}{ Anthropometrics parameters and blood pressure } \\
\hline Nutritional status [BMI] & $31.0 \pm 4.7$ & $31.0 \pm 4.9$ & $30.9 \pm 4.5$ & NS \\
\hline Underweight [n; \%] & $37 ; 0.2$ & $13 ; 0.1$ & $24 ; 0.2$ & \multirow{4}{*}{$<0.005$} \\
\hline Normal weight [n; \%] & 1,930; 9.4 & 1,090; 10.9 & $840 ; 8.0$ & \\
\hline Overweight [ $n$; \%] & 6,$548 ; 31.9$ & 3,$053 ; 30.4$ & 3,$495 ; 33.2$ & \\
\hline Obesity [n; \%] & 12,$035 ; 58.6$ & 5,$879 ; 58.6$ & 6,$156 ; 58.5$ & \\
\hline Visceral obesity $[n ; \%]$ & & $8.818 ; 87.9$ & 7,$927 ; 75.4$ & $<0.001$ \\
\hline Systolic blood pressure [mmHg] & $137 \pm 16$ & $136 \pm 16$ & $139 \pm 16$ & NS \\
\hline$>140 \mathrm{mmHg}[n ; \%]$ & 7,$938 ; 38.3$ & 3,$545 ; 34.9$ & 4,$393 ; 41.5$ & $<0.001$ \\
\hline Diastolic blood pressure [mmHg] & $84 \pm 10$ & $83 \pm 10$ & $85 \pm 11$ & NS \\
\hline$>90 \mathrm{mmHg}[n ; \%]$ & 4,$519 ; 21.7$ & 1,$819 ; 17.8$ & 2,$700 ; 25.4$ & $<0.001$ \\
\hline \multicolumn{5}{|l|}{ Clinical characteristics } \\
\hline \multicolumn{5}{|l|}{ Reason for the patient to visit } \\
\hline Obesity [n; \%] & 4,$136 ; 20.1$ & 2,$053 ; 20.5$ & 2,083; 19.8 & NS \\
\hline Type 1 diabetes [n; \%] & $336 ; 1.6$ & $153 ; 1.5$ & $183 ; 1.7$ & NS \\
\hline Type 2 diabetes [ $n$; \%] & 4,$169 ; 20.3$ & 2,078; 20.7 & 2,091; 19.9 & NS \\
\hline Hypertension [ $n$; \%] & 9,$367 ; 45.6$ & 4,$573 ; 45.6$ & 4,$794 ; 45.6$ & NS \\
\hline Dyslipidemia [n; \%] & 5,$431 ; 26.4$ & 2,$651 ; 26.4$ & 2,$780 ; 26.4$ & NS \\
\hline Ischemic heart disease [ $n ; \%]$ & 1,$301 ; 6.3$ & $444 ; 4.4$ & $857 ; 8.2$ & $<0.001$ \\
\hline Heart failure $[n ; \%]$ & $482 ; 2.3$ & $215 ; 2.1$ & $267 ; 2.5$ & NS \\
\hline Chronic liver disease $[n ; \%]$ & 3,$560 ; 17.3$ & 1,711; 17.1 & 1,$849 ; 17.6$ & NS \\
\hline \multicolumn{5}{|l|}{ Used drugs } \\
\hline ACE-I $[n ; \%]$ & 9,$983 ; 48.6$ & 4,$860 ; 48.4$ & 5,$123 ; 48.7$ & NS \\
\hline Sartan $[n ; \%]$ & 5,$651 ; 27.5$ & 2,$695 ; 26.9$ & 2,956; 28.1 & $<0.05$ \\
\hline Loop diuretic [n; \%] & 2,$876 ; 14.0$ & 1,$275 ; 12.7$ & 1,$601 ; 15.2$ & $<0.001$ \\
\hline Thiazide/thiazide-like diuretic [ $n$; \%] & 4,$583 ; 22.3$ & 2,$278 ; 22.7$ & 2,$305 ; 21.9$ & NS \\
\hline Statin $[n ; \%]$ & 11,$823 ; 57.5$ & 5,$715 ; 57.0$ & 6,$108 ; 58.1$ & NS \\
\hline Fibrate $[n ; \%]$ & 3,$188 ; 15.5$ & 1,$448 ; 14.4$ & 1,$740 ; 16.5$ & $<0.001$ \\
\hline Ezetimibe $[n ; \%]$ & $303 ; 1.5$ & $122 ; 1.2$ & $181 ; 1.7$ & $<0.01$ \\
\hline Metformin [n; \%] & 6,$354 ; 30.9$ & 3,206; 31.9 & 3,148; 29.9 & $<0.01$ \\
\hline Sulfonylurea [n; \%] & 1,$949 ; 9.5$ & $900 ; 9.0$ & 1,$049 ; 10.0$ & $<0.01$ \\
\hline Insulin $[n ; \%]$ & 1,$235 ; 6.0$ & $571 ; 5.7$ & $664 ; 6.3$ & NS \\
\hline
\end{tabular}


Table 2. Application of diagnostics procedures in accordance with European NAFLD recommendations by Polish general practitioners

\begin{tabular}{|c|c|c|c|c|}
\hline Parameters & $\begin{array}{l}\text { Study group } \\
(N=20,550)\end{array}$ & $\begin{array}{c}\text { Women } \\
(n=10,035]\end{array}$ & $\begin{array}{c}\text { Men } \\
(n=10,515)\end{array}$ & $p$ \\
\hline NAFLD diagnosis before current visit & 9,$909 ; 48.2$ & 4,$691 ; 46.7$ & 5,$218 ; 49.6$ & $<0.001$ \\
\hline \multicolumn{5}{|l|}{$\begin{array}{l}\text { Risk factors and diagnostics of NAFLD before } \\
\text { current visit [ } n ; \%]\end{array}$} \\
\hline Obesity & 1,$873 ; 45.3$ & $947 ; 46.1$ & $926 ; 44.5$ & 0.002 \\
\hline Type 2 diabetes & 2,$220 ; 53.3$ & 1,$122 ; 54.0$ & 1,$098 ; 52.5$ & $<0.001$ \\
\hline Hypertension & 4,$343 ; 46.4$ & 1,$989 ; 43.5$ & 2,354; 49.1 & 0.007 \\
\hline Dyslipidemia & 2,$503 ; 46.1$ & 1,$203 ; 45.4$ & 1,$300 ; 46.8$ & NS \\
\hline Ischemic heart disease & $738 ; 56.7$ & $236 ; 53.2$ & $502 ; 58.6$ & $<0.001$ \\
\hline \multicolumn{5}{|l|}{ Performed diagnostic test } \\
\hline Liver enzymes activity [ $n$; \%] & 9,581; 96.7 & 4,$561 ; 97.2$ & 5,$020 ; 96.2$ & $<0.001$ \\
\hline Liver ultrasound [n; \%] & 8,$563 ; 86.4$ & 3,$930 ; 83.8$ & 4,$633 ; 88.8$ & $<0.001$ \\
\hline Points scale [n; \%] & $748 ; 7.5$ & $286 ; 6.1$ & $462 ; 8.9$ & $<0.001$ \\
\hline Elastography [n; \%] & $419 ; 4.2$ & $150 ; 3.2$ & $269 ; 5.2$ & $<0.001$ \\
\hline Medical history & $(n=10,641)$ & $(n=5,344)$ & $(n=5,297)$ & \\
\hline Alcohol consumption [ $n$; \%] & 9,$708 ; 91.2$ & 4,$755 ; 89.0$ & 4,$953 ; 93.5$ & $<0.001$ \\
\hline $\begin{array}{l}\text { Patient's or family history of type } 2 \text { diabetes, } \\
\text { hypertension and cardiovascular diseases }[n ; \%]\end{array}$ & 9,$660 ; 90.8$ & 4,$876 ; 91.2$ & 4,$784 ; 90.3$ & NS \\
\hline $\begin{array}{l}\text { Body mass changes - significant weight gain or } \\
\text { loss in a short period of time }[n ; \%]\end{array}$ & 9,$334 ; 87.7$ & 4,$730 ; 88.5$ & 4,$604 ; 86.9$ & $<0.01$ \\
\hline HBV or HCV infection $[n ; \%]$ & 8,$122 ; 76.3$ & 4,$099 ; 76.7$ & 4,$023 ; 75.9$ & NS \\
\hline \multicolumn{5}{|l|}{ Recommended tests } \\
\hline Liver enzymes activity [ $n$; \%] & 11,$234 ; 54.7$ & 5,$601 ; 55.8$ & 5,$633 ; 53.6$ & 0.001 \\
\hline Liver ultrasound [n; \%] & 9,$918 ; 48.3$ & 4,$969 ; 49.5$ & 4,$949 ; 47.1$ & $<0.001$ \\
\hline $\begin{array}{l}\text { Patients diagnosed with NAFLD on the basis of } \\
\text { previously performed tests }\end{array}$ & 9,$821 ; 47.8$ & 4,$613 ; 46.0$ & 5,$208 ; 49.5$ & $<0.001$ \\
\hline $\begin{array}{l}\text { Percentage of patients with other causes of liver } \\
\text { steatosis excluded }\end{array}$ & 6,$927 ; 70.5$ & 3,$269 ; 70.9$ & 3,$658 ; 70.2$ & $<0.001$ \\
\hline Tests in NAFLD patients without diabetes & $(n=6,927)$ & $(n=3,269)$ & $(n=3,658)$ & \\
\hline Fasting glucose level $[n ; \%]$ & 5,$247 ; 75.7$ & 2,$370 ; 72.5$ & 2,877; 78.6 & $<0.001$ \\
\hline OGTT $[n ; \%]$ & 2,$681 ; 38.7$ & 1,$183 ; 36.2$ & 1,$498 ; 41.0$ & $<0.001$ \\
\hline Fasting insulin level [n; \%] & 1,$043 ; 15.1$ & $457 ; 14.0$ & $586 ; 16.0$ & NS \\
\hline HOMA-IR calculation $[n ; \%]$ & $378 ; 5.5$ & $172 ; 5.3$ & $206 ; 5.6$ & NS \\
\hline Morphology [n; \%] & 4,$922 ; 71.1$ & 2,$250 ; 68.8$ & 2,672; 73.0 & $<0.001$ \\
\hline Lipid profile [n; \%] & 4,$597 ; 66.4$ & 2,$141 ; 65.5$ & 2,$456 ; 67.1$ & NS \\
\hline Uric acid level [n; \%] & 4,$519 ; 65.2$ & 2,$119 ; 64.8$ & 2,$400 ; 65.6$ & NS \\
\hline \multirow{2}{*}{$\begin{array}{l}\text { Percentage of NAFLD patients with fibrosis assessed } \\
\text { on the basis of point scale (serous markers) }\end{array}$} & 5,$247 ; 75.7$ & 2,$370 ; 72.5$ & 2,877; 78.6 & $<0.001$ \\
\hline & 2,$681 ; 38.7$ & 1,$183 ; 36.2$ & 1,498; 41.0 & $<0.001$ \\
\hline \multirow{2}{*}{$\begin{array}{l}\text { Percentage of patients referred to the hepatology } \\
\text { clinic, in whom significant fibrosis could not be } \\
\text { excluded }\end{array}$} & $(n=7,706)$ & $(n=3,719)$ & $(n=3,987)$ & \\
\hline & 4,$726 ; 61.3$ & 2,$269 ; 61.0$ & 2,$457 ; 61.6$ & NS \\
\hline $\begin{array}{l}\text { Percentage of patients referred to the hepatology } \\
\text { clinic with performed }\end{array}$ & $(n=4,726)$ & $(n=2,269)$ & $(n=2,457)$ & \\
\hline Elastography $[n ; \%]$ & 1,$896 ; 40.1$ & $872 ; 38.4$ & 1,$024 ; 41.7$ & NS \\
\hline Liver biopsy [ $n$; \%] & $624 ; 13.2$ & $257 ; 11.3$ & $367 ; 14.9$ & $<0.001$ \\
\hline
\end{tabular}




\begin{tabular}{|c|c|c|c|c|c|c|c|c|c|c|c|}
\hline & 2 & $\stackrel{\vec{\sigma}}{\circ}$ & z & $\overrightarrow{0}$ & . & $\overrightarrow{0}$ & $\overrightarrow{0}$ & $\tilde{z}$ & $\mid \begin{array}{l}\overrightarrow{8} \\
\dot{0}\end{array}$ & $z$ & \\
\hline 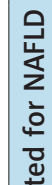 & 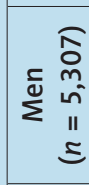 & 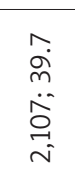 & $\begin{array}{c}\mathcal{N} \\
\dot{J} \\
\infty \\
\dot{o} \\
m \\
\sim \\
\sim\end{array}$ & 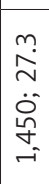 & $\begin{array}{l}\stackrel{0}{\dot{m}} \\
\dot{m} \\
\dot{0} \\
\stackrel{0}{0} \\
\rightarrow\end{array}$ & $\begin{array}{l}m \\
\text { ó } \\
\dot{g} \\
\dot{g} \\
\dot{v}\end{array}$ & $\left|\begin{array}{c}0 \\
\infty \\
m \\
\ddot{n} \\
0 \\
i \\
i\end{array}\right|$ & $\begin{array}{c}\infty \\
\dot{\sim} \\
\stackrel{\sim}{-} \\
\infty \\
\sim\end{array}$ & $\mid \begin{array}{c}n \\
m \\
m \\
\dot{0} \\
\stackrel{0}{-i}\end{array}$ & 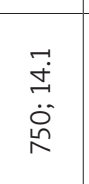 & \\
\hline $\begin{array}{l}\frac{n}{3} \\
\bar{n} \\
\underline{n} \\
\bar{c}\end{array}$ & 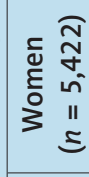 & 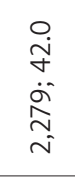 & $\begin{array}{l}0 \\
\dot{0} \\
+ \\
\dot{\delta} \\
o \\
\dot{d} \\
i\end{array}$ & 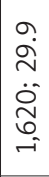 & 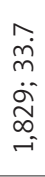 & $\begin{array}{l}0 \\
\dot{q} \\
0 \\
\dot{0} \\
0 \\
i\end{array}$ & 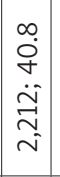 & $\begin{array}{l}a \\
\ddot{m} \\
\ddot{0} \\
\dot{0} \\
\end{array}$ & $\left|\begin{array}{c}m \\
b \\
m \\
0 \\
0 \\
\alpha \\
\rightarrow \\
-1\end{array}\right|$ & 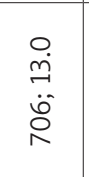 & \\
\hline$\approx$ & $\begin{array}{l}\widehat{\alpha} \\
\stackrel{1}{0} \\
0 \\
0 \\
11 \\
3\end{array}$ & $\begin{array}{l}o \\
\dot{q} \\
\dot{q} \\
\dot{\infty} \\
m \\
\dot{+}\end{array}$ & $\begin{array}{l}\vec{y} \\
\dot{\sigma} \\
\infty \\
\infty \\
\infty \\
\infty \\
\sigma \\
\sim\end{array}$ & $\begin{array}{l}0 \\
0 \\
N \\
N \\
0 \\
0 \\
\dot{m} \\
\dot{m}\end{array}$ & 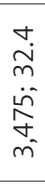 & $\begin{array}{l}\tilde{a} \\
\tilde{n} \\
\dot{\tilde{\sigma}} \\
\tilde{\sigma} \\
\tilde{f}\end{array}$ & 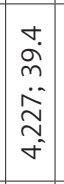 & 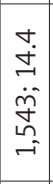 & $\mid \begin{array}{c}\infty \\
\dot{m} \\
m \\
\dot{m} \\
\hat{m} \\
m\end{array}$ & $\begin{array}{l}\stackrel{0}{0} \\
\ddot{n} \\
\ddot{b} \\
\stackrel{\leftrightarrow}{\leftrightarrow} \\
\stackrel{i}{r}\end{array}$ & \\
\hline & e & $\begin{array}{l}\overline{8} \\
\dot{0}\end{array}$ & $\begin{array}{l}\overrightarrow{8} \\
0 \\
0 \\
v\end{array}$ & 悹 & $\Sigma$ & $\begin{array}{l}\vec{\delta} \\
\dot{0} \\
\text { v }\end{array}$ & $\begin{array}{l}\overrightarrow{8} \\
\dot{0} \\
\dot{0}\end{array}$ & $\Sigma$ & $\begin{array}{l}\overrightarrow{8} \\
0 \\
0\end{array}$ & $\Sigma$ & \\
\hline $\begin{array}{l}\frac{1}{4} \\
\frac{1}{2} \\
\frac{1}{3} \\
\frac{3}{3} \\
\frac{8}{0}\end{array}$ & 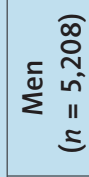 & $\begin{array}{l}\overrightarrow{⿱ ⺊ 口} \\
\infty \\
\dot{+} \\
\dot{y} \\
\dot{+}\end{array}$ & 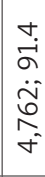 & $\begin{array}{l}m \\
\dot{b} \\
\dot{\sigma} \\
\vec{\sigma} \\
m\end{array}$ & 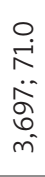 & 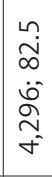 & $\begin{array}{c}m \\
\tilde{T} \\
\dot{0} \\
m \\
\dot{\sigma} \\
\dot{\sigma}\end{array}$ & $\mid \begin{array}{c}0 \\
\dot{i} \\
n \\
\ddot{j} \\
\dot{f} \\
-i \\
-i\end{array}$ & $\left|\begin{array}{c}0 \\
\dot{D} \\
\ddot{a} \\
0 \\
\dot{m} \\
\dot{n}\end{array}\right|$ & $\begin{array}{l}\overrightarrow{0} \\
m \\
\dot{0} \\
\infty \\
\infty \\
\rightarrow\end{array}$ & \\
\hline 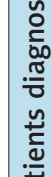 & 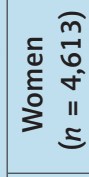 & 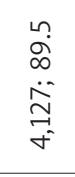 & $\begin{array}{l}m \\
\mathscr{8} \\
o \\
\dot{y} \\
\dot{J} \\
\dot{f}\end{array}$ & 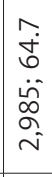 & $\begin{array}{l}\vec{N} \\
\stackrel{\sim}{\sim} \\
\tilde{N} \\
m^{-}\end{array}$ & $\begin{array}{l}0 \\
\dot{0} \\
1 \\
\tilde{\hat{\sigma}} \\
\underline{\sigma} \\
\tilde{m}\end{array}$ & $\begin{array}{l}\underset{\dot{\omega}}{\dot{\omega}} \\
\infty \\
\dot{\infty} \\
\tilde{n} \\
\hat{m}\end{array}$ & 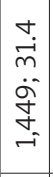 & 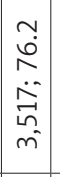 & $\begin{array}{l}a \\
\omega \\
m \\
\dot{0} \\
\hat{\omega} \\
\stackrel{r}{r}\end{array}$ & \\
\hline$a$ & $\begin{array}{l}\widehat{I} \\
\infty \\
0 \\
11 \\
3\end{array}$ & $\begin{array}{l}\stackrel{\sim}{\infty} \\
\infty \\
\dot{\vec{b}} \\
\stackrel{0}{\infty} \\
\infty\end{array}$ & $\begin{array}{l}\hat{n} \\
\text { o } \\
\ddot{b} \\
o \\
\alpha \\
\sigma\end{array}$ & 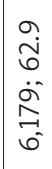 & 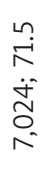 & $\begin{array}{l}\hat{0} \\
\infty \\
\ddot{\tilde{N}} \\
\tilde{N} \\
\end{array}$ & 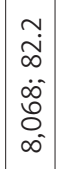 & 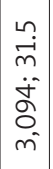 & $\begin{array}{c}\stackrel{v}{x} \\
\\
\ddot{\infty} \\
0 \\
N \\
N\end{array}$ & $\begin{array}{l}0 \\
\dot{\omega} \\
\ddot{0} \\
\hat{n} \\
\stackrel{n}{n}\end{array}$ & \\
\hline & & 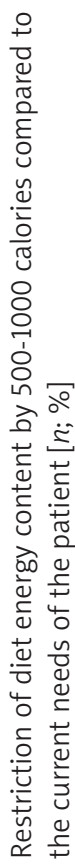 & 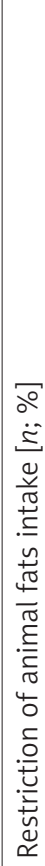 & 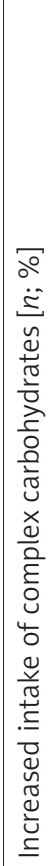 & 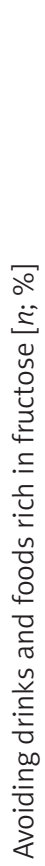 & 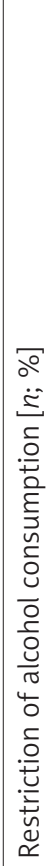 & 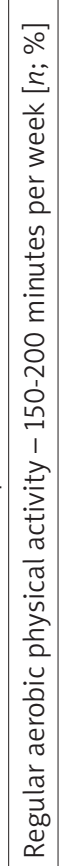 & 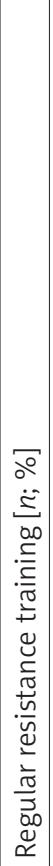 & 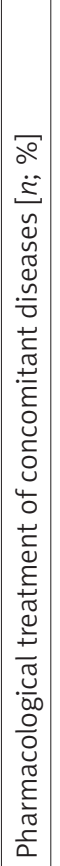 & 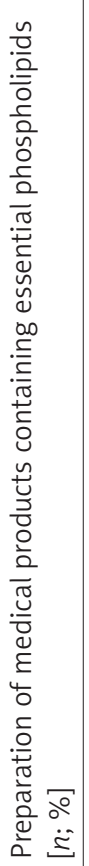 & 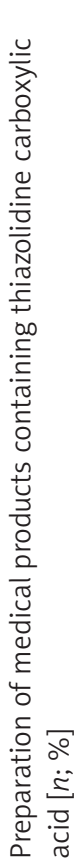 \\
\hline
\end{tabular}

- pharmaceutical products containing a thiazolidine carboxylic acid in $74.9 \%$ of patients with NAFLD and 29.2\% suspected for NAFLD (Table 3 ).

\section{Discussion}

As mentioned above, the cause of the primary form of NAFLD is obesity, and fatty liver is one of the key factors in the pathogenesis of type 2 diabetes. Therefore, in 2016 the European Association for the Study of the Liver (EASL), the European Association for the Study of Diabetes (EASD) and the European Association for the Study of Obesity (EASO) formulated common recommendations for diagnosis and treatment of NAFLD in the primary health care setting. From 1990 year by year various scientific associations were releasing recommendations for primary care practitioners. However, the implementation of these guidelines was rarely assessed in daily clinical practice. Our study is the first to assess implementation of EASL, EASD and EASO recommendations in Poland.

Despite the fact that all patients included in this study were reported with diseases described as risk factors of NAFLD, including 90\% with overweight and obesity, diagnostic work-up for the disease, prior to the current visit, was performed in less than half of the patients. This shows discrepancies with the established recommendations [6], according to which all these patients should be screened for NAFLD. Thus, the obtained results indicate that Polish general practitioners too rarely perform screening for NAFLD in patients with the highest risk of developing the primary form of the disease [4]. More often doctors screened for NAFLD patients with complications of obesity such as type 2 diabetes and ischemic heart disease. This means that diagnostics of NAFLD in Poland is performed quite late.

According to the recommendation [6], activity of GOT and GTT and liver ultrasonography were used for NAFLD screening. It should be noted that NAFLD was diagnosed in less than half of the screened patients, among whom in $70 \%$ secondary causes of liver steatosis were excluded. Concerning nutritional status of the study population, this diagnosis was less frequent than in the other overweight and obese populations (70-80\%) [2] but slightly higher than in the general population (17-33\%) [1]. This suggests that NAFLD screening should be repeated especially in patients with weight gain and after development of obesity complications.

The guidelines recommend performance of anamnesis to exclude other causes of liver steatosis [6]. Despite the fact that in all patients not screened for NAFLD before, there were indications for screening implementation, not everyone was asked about alcohol consumption, significant weight loss or gain in a short time and HBV or 
$\mathrm{HCV}$ infections, and the occurrence of type 2 diabetes, hypertension and cardiovascular diseases in the patients' family. In addition, only about half of this population received a recommendation for determination of liver enzymes activity and ultrasonography, despite the fact that indications for this screening were present in almost all those not previously examined.

The assessed recommendation in patients with NAFLD without diagnosis of type 2 diabetes suggests assessment of insulin resistance using HOMA-IR and screening for type 2 diabetes [6]. Polish general practitioners rarely followed this recommendation. Fasting plasma glucose was determined in more than three quarters of this group and the OGTT was performed in less than $40 \%$ and HOMA-IR was calculated in only about $6 \%$ of this population. These results suggest poor knowledge of current and older recommendations, as well as the role of NAFLD in the pathogenesis of type 2 diabetes.

According to the assessed recommendation in the high risk group (age above 50 years, type 2 diabetes and/ or other components of metabolic syndrome) active detection of NASH with fibrosis should be undertaken [6]. Despite the fact that a significant percentage of patients had high risk of NASH with fibrosis, only slightly more than $20 \%$ of patients diagnosed with NAFLD were evaluated for fibrosis based on a point scale. In addition, only $60 \%$ of patients with suspected significant fibrosis were referred to a hepatology outpatient clinic. It is interesting that elastography was performed only in $40 \%$ of patients referred to a hepatology outpatient clinic and liver biopsy in less than $15 \%$. On the basis of our data it is not known whether liver biopsy was performed as the first study or according to recommendations to confirm significant fibrosis [6].

The second part of EASL, EASD and EASO recommendation concerns treatment of NAFLD. The basic procedure are changes of eating habits causing $7-10 \%$ weight loss and qualitative changes in food and drinks [6]. It should be noted that in the present study a substantial proportion of patients with NAFLD did not receive recommendations concerning changes of eating habits. More often, reduced consumption of animal fat and increased consumption of complex carbohydrates were recommended. Much less frequently changes of eating habits were recommended to patients with suspected NAFLD. It may indicate the lack of general practitioners' knowledge about the impact of macronutrients on development of NAFLD and the lack of knowledge on current recommendations, and perhaps the lack of motivation and time for patient counseling in this aspect. These data show the importance of the role that should be played by dieticians and dietary advice in primary care settings, still not covered by the national health fund in Poland.
According to the recommendations the second element of NAFLD treatment is regular physical activity: 150-200 minutes of aerobic physical activity per week of moderate intensity and resistance training [6]. Regular aerobic physical activity was recommended in about $80 \%$ and resistance training in $30 \%$ of patients diagnosed with NAFLD and patients suspected with NAFLD. This may indicate a lack of knowledge not only of the current NAFLD recommendations, but also of insufficient knowledge about the role of physical activity, especially in treatment of obesity and its complications. It should also be noted that both changes of diet and increased physical activity are not only part of NAFLD therapy but also of overweight and obesity management. Therefore, both may have the same barriers including attitudes toward obese patients, lack of insurance payments for obesity care, poor education during medical school and postgraduate training and lack of time $[9,10]$. It has also been shown that physician have lower respect for obese patients and this adversely affects the quality of care [11]. In addition, another study has shown that primary care practitioners more often expressed interest in obese patients' referral to a specialist [12]. There is a lack of studies assessing attitudes of doctors towards obese patients in Poland. Therefore, it is difficult to state how they could have an impact on the implementation of the recommendations. Further studies are necessary to assess this aspect.

The third recommended element of NAFLD treatment is the pharmacological treatment of obesity-related diseases [6]. This element of treatment was also implemented in only about $70 \%$ of patients diagnosed with NAFLD and about $30 \%$ of patients suspected with the diagnosis. The obtained results neither allow the causes of such therapeutic decisions to be explained nor rule out the possibility that some patients had started the pharmacotherapy earlier.

However, despite the fact that guidelines do not recommend the use of essential phospholipids and thiazolidine carboxylic acid in the treatment of NAFLD, they were frequently used in patients with NAFLD and less often in patients with suspected NAFLD. The factors explaining these therapeutic decisions and the efficacy of this treatment should be assessed in the future.

\section{Conclusions}

Polish general practitioners too rarely perform diagnostic tests for NAFLD and recommend changes in diet and physical activity, and too rarely perform tests for carbohydrate metabolism disturbances.

Diagnostic workup and NAFLD therapy are in line with the current European guidelines. 


\section{Acknowledgments}

The study was carried out as a research project supported by a scientific grant of Valeant sp. $z$ o.o. sp.j. organized by Europharma M. Rachtan sp. z o.o. Poland.

\section{Disclosure}

The authors report no conflict of interest.

\section{References}

1. Lee RG. Nonalcoholic steatohepatitis: a study of 49 patients. Hum Pathol 1989; 20: 594-598.

2. Reid AE. Nonalcoholic fatty liver disease. In: Feldman M, Friedman LS, Brandt LJ (eds.). Sleisenger and Fordtran's gastrointestinal and liver disease. 8th ed. Saunders, Philadelphia 2006; 1793-1805.

3. Orlik B, Handzlik G, Olszanecka-Glinianowicz M. Rola adipokin i insulinooporności w patogenezie niealkoholowej stłuszczeniowej choroby wątroby. Postepy Hig Med Dos 2010; 64: 212-219.

4. Machado M, Cortez-Pinto H. Non-alcoholic fatty liver disease and insulin resistance. Eur J Gastroenterol Hepatol 2005; 17: 823-826.

5. McCullough AJ. Pathophysiology of nonalcoholic steatohepatitis. J Clin Gastroenterol 2006; 40 (Suppl. 1): S17-S29.

6. European Association for the Study of the Liver (EASL), European Association for the Study of Diabetes (EASD) and European Association for the Study of Obesity (EASO) (2016) EASLEASD-EASO Clinical Practice Guidelines for the management of non-alcoholic fatty liver disease. J Hepatol 2016; 64: 1388-1402.

7. Gundermann KJ, Gundermann S, Drozdzik M, Mohan Prasad VG. Essential phospholipids in fatty liver: a scientific update. Clin Exp Gastroenterol 2016; 9: 105-117.

8. Farbiszewski R, Radecka A, Chwiecko M, Holownia A. The effect of heparegen on antioxidant enzyme activities in ethanol-induced liver injury in rats. Alcohol 1992; 9: 403-407.

9. Forman-Hoffman V, Little A, Wahls T. Barriers to obesity management: a pilot study of primary care clinicians. BMC Fam Pract 2006; 7: 1-11.

10. Kushner RF. Barriers to providing nutrition counseling by physicians: a survey of primary care practitioners. Nutr Clin Pract 2010; 25: 502-509.

11. Huizinga MM, Cooper LA, Bleich SN, et al. Physician respect for patients with obesity. J Gen Intern Med 2009; 24: 1236-1239.

12. Kristeller JL, Hoerr RA. Physician attitudes toward managing obesity: differences among six specialty groups. Prev Med 1997; 26: 542-549. 